\title{
PC-ASTAKINE MRNA LEVELS DECREASE AFTER BOTH LPS INJECTION AND HEMOLYMPH WITHDRAWALS IN THE GASTROPOD PEST POMACEA CANALICULATA
}

\author{
A Accorsi ${ }^{1,2}$, K Gotting ${ }^{1}$, E Ross ${ }^{1,2}, \mathrm{~S}$ Benatti $^{3}, \mathrm{M} \mathrm{Nasi}^{4}$, E Ottaviani ${ }^{3}$, A \\ Sánchez Alvarado ${ }^{1,2}, \underline{D}$ Malagoli $^{38}$ \\ ${ }^{1}$ Stowers Institute for Medical Research, Kansas City, MO, USA \\ ${ }^{2}$ Howard Hughes Medical Institute, Stowers Institute for Medical Research, \\ Kansas City, MO, USA \\ ${ }^{3}$ Department of Life Sciences, University of Modena and Reggio Emilia, \\ Modena, Italy \\ ${ }^{4}$ Department of Surgery, Medicine, Dentistry and Morphological Sciences, \\ University of Modena and Reggio Emilia, Modena, Italy
}

The freshwater gastropod Pomacea canaliculata has recently been included among emerging pests in the EU. Hence, its immune system may be a promising target for pest control strategies. By analyzing a set of $P$. canaliculata transcriptomes, we have discovered an Astakine-1-like molecule ( $P c$-Astakine) in this organism. Astakine was first isolated as a hematopoietic cytokine in the crayfish Pacifastacus leniusculus (Pl-Astakine-1), and later found in arthropods and the bivalve Crassostrea gigas. Bioinformatic analyses show that $P c$-Astakine is a 121 aa protein with a conserved distribution pattern of cysteines retrievable in both $\mathrm{Pl}$-Astakine-1 and the vertebrate Prokineticin protein family. Pc-astakine is expressed at low levels in circulating hemocytes, but markedly in hemocyte reservoirs (e.g., ampulla) and hematopoietic tissue (pericardial fluid) suggesting a conserved role in hematopoiesis. We tested this potential role of Pc-Astakine in two ways. First, we measured RNA levels of this gene in immune-related tissues $24 \mathrm{~h}$ after injection of $50 \mu \mathrm{g}$ of Escherichia coli-derived lipopolysaccharide (055:B5) (LPS). We observed a sharp decrease of Pc-astakine mRNA in all the analyzed organs, with Pc-astakine mRNA levels almost undetectable in anterior kidney (a hemocyte reservoir) when compared to sham-injected controls. Second, we defined the profile of Pc-astakine expression after a series of 4 hemolymph withdrawals performed within $72 \mathrm{~h}$. As with the LPS-treatment, a general decrease of mRNA levels was observed, with the largest decrease observed in hematopoietic tissue and circulating hemocytes (reduced to $20 \%$ of the control). Altogether, we hypothesize that the decrease of Pcastakine mRNA after both LPS injection and hemolymph withdrawal may be due to an increased rate of translation, rather than a reduction of gene expression. This is in part supported by similar observations in the oyster $C$. gigas in which massive translation of $C g$-astakine is observed as a consequence of environment stress, including immune stimuli, and in P. leniusculus in which increased levels of Astakine in the plasma of LPS-injected animals have been related to an increased translational rate.

To our knowledge, this is the first report of an astakine-like molecule in gastropods and our data indicate a potential involvement of $P c$-Astakine in both the immune response and hematopoiesis of the molluscan pest $P$. canaliculata. 
Keywords: mollusk; pest; hematopoiesis; transcriptome; cytokine

${ }^{\S}$ Corresponding author. Tel.: +39 0592055538; Fax: +39 0592055548.

E-mail address: davide.malagoli@unimore.it 